\section{Ueber einige Herzgifte.}

Vortrag gehalten im Verein für innere Medicin. Von

\section{Dr. Ferdinand Karewski,} pract. Arzt in Berlin.

Meine Herren! Das Studium der Herzgifte ist ein so beliebtes und so vielfach bearbeitetes Thema, dass jeder neue Untersucher fast der Entschuldigung bedarf, wenn er es unternimmt, die Ergebnisse seiner Experimente an die Oeffentliclkeit zu bringen.

Aber gerade dieser Ueberfluss an einschlägigen Arbeiten ist vielleicht ein Beweis dafür, wie wenig feststehend das bisher Bekannte ist, und wie dringend es einer Bestätigung resp. Berichtigung bedarf.

Der Grund hiervon liegt auf der Hand.

Die Unterhaltung und Regelung der Herzbewegung ist bekanntlich unterthan dem Regiment zweier pervöser Apparate: demjenigen im Centralnervensystem und dem, welches in der Herzsubstanz selbst gelegen ist. Letzteres dirigirt wiederum nicht das ganze Herz, sondern nur die Vorhöfe und die Herzbasis, während der grössere Theil der Herzkammern frei ist von automatischen Nervencentren. So giebt es schon 3 Factoren, die bei der Betrachtung des Einflusses von Giften auf sie von einander gesondert werden müssen. - Dazu kommt noch als vierter, dass die Herzgifte vielleicht auch die Gefässe selbst alteriren, ein Umstand, der naturgemäss eine Aenderung der Herzthätigkeit im Gefolge haben muss, da diese sich ja je nach dem wachsenden oder verringerten Widerstand im Stromgebiet regelt.

Als ich demnach vor inehr als Jahresfrist von den Herren Geh. Rath L eyden und Dr. A. Fränkel aufgefordert wurde, einige klinisch wichtige Substanzen, die Digitalis, das Veratrin und das Kali nitricum bezüglich ihrer Einwirkung auf das Centralorgan des Gefässsystems zu prüfen, wurde gleich von vorn herein in Aussicht genommen, dies so zu thun, dass 2 Factoren, die extracardialen Centra und das Gefässsystem ausgeschaltet würden, und die beiden anderen, die intracardialen Centra und die Muskelsubstanz von einander getrennt betrachtet wurden.

Solches aber ist möglich durch Anwendung einer Untersuchungsmethode, die schon lange Jalıre in der Physiologie gebräuchlich ist, und der wir zum nicht geringen Theil unsere Kenntniss von den complicirten Mechanismen verdanken, welche den Bewegungsapparat des Herzens darstellen. Ich meine die Anwendung des Froschherzens, das ja den Tod des Thieres Stunden lang überlebt und das wir von Ludwig ${ }^{1}$ ) gelernt haben, mit einem künstlichen Kreislauf zu versehen, welcher zugleich gestattet, die Herzbewegungen am Kymographion aufzeichnen zu lassen.

Dieser künstliche Kreislauf nach der ursprünglichen Angabe des Erfinders im Wesentlichen aus einer Mariolte'schen Flasche und einem kleinen Quecksilbermanometer mit Zeichenfeder bestehend, ist von den späteren Untersuchern, je nach dem Bedürlniss des Einzelnen, vielfach modificirt worden.

Ich selbst gebrauchte zur Durchspülung des Herzens mit der Nährflässigkeit, den kürzlich von Williams) angegebenen Apparat mit einigen kleinen eigenen Modificationen.

1) Arbeiten des physiologischen Instituts zu Leipzig, 1866-1875.

2) Arch. f. experim. Pharm. u. Path. Bd. XIII.
Von einem Speisungsgefäss führt ein Kaoutchoucschlauch zu einem kleinen Ventil, das nur Vorwärtsbewegung der Nährflüssigkeit gestattet; dieser mündet im Herzen. Aus letzterem führt ein gleiches Ventil, das wiederum den Räckfluss hindert, zu einem Quectisilbermanometer, vor welchem sich eine Abflussöffnung für die verbrauchte Flüssigkeit befindet.

Dieselbe kann durch einen Quetschhahn so eingestellt werden, dass das Quecksilber im Manometer unter stets gleichem Druck bleibt.

Zur Ernährung des Herzens diente frisches, defibrinirtes Kaninchenblut in geeigneter Weise mit 0,6 procentiger Koclisalzlösung verdünnt. Um das Herz ferner vor Vertrocknung zu sclützen, wurde es in ein durchsichtiges Glasgefäss versenkt, das, mit Salzwasser gefüllt, gestattete, die Herzbewegungen mit den Augen zu controlliren.

Jedes Gift wurde zunächst auf das nervenhaltige, intacte, und dann auf das seiner Nervencentra durch feste Umschnürung unterhalb der Atrioventricularfurche beraubte geprüft.

Ich beginne mit demjenigen Präparat, welches das meiste Interesse des Arztes besitzt, der Digitalis

Von keinem Herzgift gilt wohl mehr das vorher Gesagte, als gerade von diesem. Es existiren zahllose Arbeiten über Digitalis und ihre Wirkung ist bekannter als die irgend eines anderen Medicaments. Dennoch sind die Autoren sich nicht über dieselbe einig. Wird von den Einen vor Allem der Einfluss auf die nervösen Elemente hervorgehoben, so legen Andere im Gegentheil das grössere Gewicht der Veränderung der Musculatur bei, die der rothe Fingerhut hervorruft. Lind in der That möchte auch ich den Letzteren Recht geben.

Denn ich fand, dass die Digitalis den Herzmuskel in einen $\mathrm{Zustand}$ erhöltten Contractionsbestrebens versetzt, der mit dem Beginn der Digitaliswirkung eintritt, unablässig zunimmt und schliesslich in einen Zustand eigenthümlicher Starre übergeht, während die Vorhöfe auclı dann noch pulsationsfälig sind.

Das einfachste und reinste Bild dieser Veränderung der contractilen Substanz erhält man, wenn man die Herzspitze, d. h. das unterhalb der Atrioventricularfläche abgeschnürte Herz alleın unter den Einfluss der Digitalis versetzt. Dann beginnt das Herz mit dem Augenblick, wo die Digitalis lange genug mit demselben in Berührung gewesen ist, um ihre verderbliche Wirkung zu äussern, sich zu contrahiren. Die Pulse werden kleiner, dadurch, dass keine rollkommenen diastolischen Erschlaffungen mehr zu Stande hommen. Die Contraction schrettet weiter fort, die diastolischen Erweiterungen werden immer geringer und schliesslich stelit das Herz in completer und permanenter Sistole still. Die aneinander gelegten Wände des Ventrikels lassen sich durch den stärksten Flüssigkeitsdruck nicht mehr ausdehnen; auch fehlt jede Reaction auf andere Reize.

Das Herz ist starr.

In der Curve drückt sich das etwa so aus: würde man die Spitzen sämmtlicher Systolen durch eine gerade Linie rerbinden und ebenso die diastolischen Abfälle durch eine Gerade bezeichnen, so verhefe die letztere zur ersteren schräg aufsteigend.

Sehr viel complicirter und mannigfaltiger gestaltet sich der Ausdruck der Digitaliswirkung an dem ganzen, intacten Herzen. Da kommt es gewissermaassen zu einem Streit zwischen zwei Gewalten, ron denen die eine das ihrer Oberholıeit unterstellte Organ zu seıner normalen Thätigkeit anlıalten will, während die andere, feindselige, es zu einer einseitigen Thätigkeit $\mathrm{zwingt,}$ deren alleinige Ausführung seine gewohnte Function unterbrechen müsste, - zur permanenten, sustolischen Contraction.

Ist aber auch die Ursache der veränderten Herzbewegung immer die gleiche, so ist diese selbst doch überreicb an Variationen. Bei kleınen Giftdosen bemerkt man zunächst eine eigenthümliche Veränderung der Ventrikeldıastole. Dieselbe wird auf halber Höhe von einer rudimentären Systole unterbrochen, so dass die Curve exquisit dicrotisch wird. Sehr häufg konımt es auch zu zwei systolischen Unterbrechungen; gleiclızeitıg sielıt man das Herz mit vermelirter Energie sich zusammenziehen.

Spricht sich schon in diesen unvollkommenen diastolischen Abfällen und der grösseren kraft, mit der die Systole ausgefülırt wird, das erhöhte Contractionsbestreben aus, so wird dasselbe noch viel augenscheinlicher, wenn man grössere Gaben Digitalis anwendet. Dann werden die Wände des Herzens starrer und der Flüssigkeitsdruck, welcher sonst die geregelte Herzthätigkeit unterhält, reicht nicht mehr dazu aus. Nicht nehr eine einzelne Vorhofsystole vermag genügende Flüssigkeitsmengen in den Ventrikel zu pumpen, sondern mehrere, an Zahl immer steigernde, werden erfordert. Naturgemäss gelangt dadurch mehr Flüssigkeit ins Herz, und da der Ventrikel sich stets bis zum Extrem zusammenzieht, so wird die Pulsation immer höher. Ebenso natürlich ist, dass die Pulsation selbst verlangsamt wird, und dass - nach einem überall in der Physiologie des Herzens gültigen Gesetz, nach welchem ein Reiz. der eine Pulsation auszulösen vermochte, für nachfolgende, wenn auch 
schwächere die Erregung des Herzens erleichtert - eıne sanze Anzahl von Pulsen einer Pause folgen, dass also Gruppenbildung eintritt.

Je mehr aber das Contractionsbestreben zunimmt, desto mehr überwiegt es auch die Nerveneinflüsse. Die Pausen werden länger, die Pulsperioden kürzer. Dazu gesellt sich dann eine fort und fort steigende Contractıon in der Pause, die auch während des Pulsirens nicht mehr völlig gelöst wird, so dass also die Pulse selbst kleiner werden - und schliesslich entsteht ein ganz merkwürdiges Phänomen: das Herz 1 st in der Pause systoliseh contrahirt und beginnt mit einer Diastole zu schlagen. Das dauert dann bis zum gänzlichen Stillstand, der nach und nach eintritt, zuerst an der Herzspitze, während Vorhöfe und Ventrikelbasis noch pulsiren. Am Ende tritt allgemeiner Stillstand en bei extremster, systolischer Contraction des Ventrikels und äusserster, diastolischer Dilatation der Vorhöfe. - Der Einfluss der Digitalis ist Sieger geblieben über denjenigen der Nervenelemente, - jedoch olne diese zu tödten. Denn sobald man die Vorhöfe von dem colossalen Druck entlastet, unter dem sie stehen, weil sie ihren Inhalt nicht mehr in den Ventrikel auspumpen konuten, fangen sie wieder an zu schlagen. Der Ventrikel selbst bleibt in der alten Zusammengezogenheit stehen, und alle Reize erweisen sich auf ihn wirkungslos.

Eine andere Form der Digitaliswirkung gestaltet sich so:

Nach elnigen wenigen Pulsen mit systolisch modificirten Diastolen contrahirt sich das Herz ganz plötzlich ziemlich erheblich und pulsirt dann in starker Pulsbeschleunıgung und mit kleinen Pulsen. Die Contraction nimmt zu, die Pulse nehmen ab und werden schliesslich so klein, dass sie keinen Ausschlag mehr im Manometer geben. Erhöht man jetzt den Druck, unter dem die Nährflüssigkeit zuströmt, um ein Weniges, so werden sofort hohe kraftvolle Pulsationen von durchaus regelmässigem Charakter ausgeführt. Jedoch es dauert nicht lange, so hat das Herz sich wieder contrahirt, und man muss einen noch stärkeren Druck einwirken lassen.

Dieser befähigt nun das Herz zu langdauernder Pulsation, in der das Contractionsbestreben aber höchst sonderbare Abweichungen von der Norm verursacht. Ausser den vorher beschriebenen Diastolen kommen solche vor, die durch 3,4 und mehr rudimentäre Systolen unterbrochen werden, so dass vielgipflige Pulse entstehen.

In dem nächsten Stadium verschwınden die vielgipfligen Pulse, aber die Pulsfolge wird verändert. Wir laben eine Art Gruppenbildung, die jedoch umgekehrt wie vorher das Contractionsbestreben des Ventrikels nıcht in der Pause, sondern in der Pulsperiode zelgt. Das reichlich mit Blut gefüllte Herz macht enen sehr hohen Puls, dann eine Diastole von etwa $2 / 3$ der Grösse, die es machen müsste, um die Abscisse zu erreichen, dann bleıbt es in dieser Weise contrahirt und führt eine Reihe von Pulsen von gleicher Höhe und Folge, deren Systolen nicht die Höhe der ersten erreichen, weil ein Theil des Ventrikelinhaltes ja ausgepumpt ist, und der contrahirte Ventrikel nicht mehr so reichlich gefüllt werden kann, deren Diastolen aber bis zur Tiefe der ersten herabsinken. Der letzte Puls hat dann eine sehr prolongirte, complete Diastole, während welcher der Ventrikel durch mehrfache Vorhofssystolen wieder sehr stark mit Blut gefüllt und so zu neuer, besonders hoher Kraftleistung befähigt wird.

Ueberhaupt hat bis zu diesem Stadıum die Arbeitsfähigkeit des Herzens immer zugenommen, so dass Schläge von doppelter Höhe der Normalpulse ausgeführt werden. Die steigende Contraction oder Starre des Ventrikels lässt nun aber die Pulse wieder mehr und mehr an Höhe abnehmen, auch Pausen treten ein, während deren es zu keiner vollen Erschlaffung kommt, und das Ende vom Spiele ist, dass auch hier systolische Pausen entstehen, mit Pulsperioden, deren paradoxer Anfang wieder eine Drastole ist.

Ziehen wir das Facit aus diesen Beobachtungen, so werden wir sagen müssen, dass die Digitalis in ihrer Anfangswirkung die Arbeitsfähigkeit des Herzmuskels erlöht durch eine Vermehrung seiner Elasticität, dass die letztere aber übergeht in eine Art von Starre, die erst eine geordnete Pulsation unmöglich macht, dann die Pulsation überhaupt aufhebt und dieses zu einer Zeit, wo die Herzsubstanz noch nicht abgestorben sein kann, da die Vorhöfe, die mit ihr gleichen Einflüssen ausgesetzt waren, noch ihre Function zu verrichten im Stande sind.

Ene ganz ähnliche Wirkung hat das Veratrin. Auch dieses erzeust systolischen Herzstillstand, und dieser kommt ebenso wie bei der Digitalıs durch ein zunehmendes Contractionsbestreben zu Stande.

Beginnen wir wieder mit dem Einfachsten, der Herzspitze, so sehen wir sofort unter dem Einfluss des Giftes die Systolen steil ansteıgen, in systolischer Contraction das Herz einige Secunden verharren und sich dann allmällig, häufig Absat $L$ - und Ruckweise wieder erweitern.

Gerade im Anfang der Wirkung dauern die systolischen Stillstände besonders lang, bis zu 10 Secunden, und werden hon und wieder von frustranen Versuchen zu diastolischer Ausdehnung unterbrochen. Es kann hier von einer geregelten Herzthätigkeit nicht die Rede sein. Bald aber ändert sich das. Die systolischen Erhebungen und Stillstände sind dann meist alle von gleicher Höhe und Dauer, und auf jede volle Systole folgt eine volle Diastole, so dass eine durchaus regelmässige Pulsation freilich von höchst barockem Aussehen vorhanden ist. Die Pulsation hört wieder ganz allmählig auf in Folge der steigenden Contraction des Ventrikels, sobald permanente systolische Stellung erreicht ist.

Hat man es mit dem ganzen Herzen zu thun, so sind 2 Arten der Veratrinwirkung zu unterscheiden. Gemeinsam haben beide, dass die Systole mit gesteigerter Energie ausgeführt wird und die Erschlaffungswelle des Herzens sich verlängert. Aber in dem einen Falle finden wir die dauernde, Tetanus-ähnliche Zusammenziehung ausgeprägter, in dem anderen kommt erhöhte Arbeitsleistung zur Beobachtung.

In dem ersten Falle ist de Pulscurve wenig verschieden von derjenigen der Herzspitze. Nur dauern hier die Stillstände noch viel länger, und dem definitiven Stillstand geht ein bis 30 Minuten währendes Stadium voraus, in welchem zwar extremste Contractionsstellung vorhanden ist, die aber durch partielle Diastolen an einzelnen Theilen des Ventrikels bald an der Spitze, bald an der Basis, bald an der linken, bald an der rechten Ventrikel wand unterbrochen wird. Manchmal kommt es auch bei dieser Art der Veratrinwirkung zu Gruppenbildung, dadurch dass nach einer Anzahl von Pulsen Pausen eintreten, während deren das Herz sich ganz allmählig so weit contrahirt, dass die Systole bei Beginn der Pulsation fast erreicht ist, und nur eine ganz geringe, schnell erfolgende Zusammenziehung dieselbe complet macht.

Die andere Form der Veratrinwirkung stellt sich, wie folgt, dar. Ganz plötzlich nimmt die Energie der Contraction zu. Die Systole steigt steil an, die Diastole geht nach und nach vor sich; während derselben pumpen die Vorhöfe durch mehrfache Contractionen den Ventrikel voll. Immer steiler und höher werden die Systolen des Ventrikels, und immer zahlreicher werden die der Vorhöfe während der Ventrikeldiastole, so dass wohl $9 \mathrm{u}$. m. erfolgen. So erreicht die Pulsation oft den vierfachen Ausschlag des Normalpulses. Natürlich nimmt die Frequenz gleichzeitig ab, der Einzelpuls entspricht dem Effect unehrfacher normaler Pulse und beansprucht den zeitlichen Verlauf ihrer Summe für sich allein. Hat die Herathätigkeit ilıren Culminationspunkt erreicht, so beginnt das Contractionsbestreben sich im entgegengesetzten Sinne zu āussern. Das Herzvolumen wird wieder kleiner und wenıger erweiterungsfähig, die Pulshöhe nimmt ab, und es entsteht die auch bei der Digitalis beobachtete Gruppenbildung von grossen und kleinen Pulsen.

Das Ende ist in allen Fällen Stillstand in Systole. Die Vorhöfe pulsiren von Neuem, wenn man aus ihnen das reichlich angesammelte Blut entleert.

Wir haben also auch beim Veratrinherzen die Aenderung der Herzbewegung als eine Muskelalteration anzuselien.

Einen vollkommenen Gegensatz zu den Wirkungen der Digitalis und des Veratin bilden die des Kali nitricum.

Bei grossen Dosen des Giftes steht sowohl das mit seinen Ganglien versehene, intacte als auch das nervenlose Herzpräparat gauz plötzlich wie mit einem Schlage in Diastole still; also auch hier kann ohne Vermittlung der Nervenelemente momentane Muskellähmung eintreten; nur nehmen gleichzeitig die Vorhöfe, d. h. die Nervenelemente an der Lähmung Theil. Stıllstand in Diastole tritt, - freilich ganz allmälig -, auch bei kleinen Dosen ein, an dem wiederum die Vorhöfe participiren. Vorhöfe wie Ventrikel, ob mit ob ohne Nerven, schlagen wieder, wenn man den Druck der Nährflüssigkeit herabsetzt, d. h. die Elasticität des Herzmuskels, welche durch Digitalis und Veratrin eine Vermehrung erfährt, wird durch Kal. nitric. herabgesetzt.

Aus allen diesen Versuchen über Digitalis, Veratrin und Kali nitricum geht also hervor, dass diese Herzgifte dıe Functionsfähigkeit des Herzmuskels zerstören durch eine Veränderung seiner normalen Elasticitätszustände.

Wie weit es übrigens richtig ist, die Erfahrungen am künstlich circulirten Froschherzen auf das intacte Herz des Warmblïters zu übertragen, darüber ist es schwer ein bestimmtes Urthell abzugeben. Die Analogie liegt gewiss sehr nahe, und es wird unter allen Umständen gerathen sein, bei der therapeutischen Anwendung dieser Gifte darauf Rücksicht zu nehmen. -

Herrn Geheimrath Leyden und Herrn Dr. A. Fränkel kann ich nicht umhin, auch von dieser Stelle aus meinen wärmsten Dank für die gütıge Lnterstūtzung, die sie mir bei Anstellung meiner Versuche zu Theil werden liessen, auszusprechen. 